\title{
FOURIER MULTIPLIERS AND INTEGRO-DIFFERENTIAL EQUATIONS IN BANACH SPACES
}

\author{
VALENTIN KEYANTUO AND CARLOS LIZAMA
}

\begin{abstract}
Operator-valued Fourier multiplier theorems are used to establish maximal regularity results for an integro-differential equation with infinite delay in Banach spaces. Results are obtained under general conditions for periodic solutions in the vector valued Lebesgue and Besov spaces. The latter scale includes in particular the Hölder spaces $C^{\alpha}, 0<$ $\alpha<1$.
\end{abstract}

\section{INTRODUCTION}

We consider the following integro-differential equation with infinite delay

$$
\left\{\begin{array}{l}
u^{\prime}(t)=A u(t)+\int_{-\infty}^{t} a(t-s) A u(s) d s+f(t), 0 \leq t \leq 2 \pi \\
u(0)=u(2 \pi) .
\end{array}\right.
$$

We will examine this problem in various spaces of $2 \pi$ - periodic vectorvalued functions: $L^{p}(0,2 \pi ; X)$ (Lebesgue-Bochner spaces), $C^{\alpha}(0,2 \pi ; X)$ (Hölder spaces), $B_{p q}^{s}(0,2 \pi ; X)$ (Besov spaces). Here $X$ is a Banach space, $A: D(A) \subset X \rightarrow X$ is a (not necessarily densely defined) closed linear operator and $f$ is an $X$-valued function defined on $[0,2 \pi]$. We note that equation (1.1) may be rewritten as

2000 Mathematics Subject Classification. Primary 45N05; Secondary 45D05, 43A15, $47 \mathrm{D} 99$.

The first author is supported in part by Convenio de Cooperación Internacional (CONICYT) Grant \# 7010675 and the second author is partially financed by FONDECYT Grant \#1010675 . 


$$
\left\{\begin{array}{l}
u^{\prime}(t)=A u(t)+\int_{0}^{\infty} a(s) A u(t-s) d s+f(t), 0 \leq t \leq 2 \pi \\
u(0)=u(2 \pi) .
\end{array}\right.
$$

Equations of this kind arise, for example, in the study of heat flow in materials of fading memory type as well as some equations of population dynamics. For more information on this subject see the papers $[8,15,11]$ and the monograph [16] (particularly Chapter II, Section 9) and the references therein. We also note the recently published paper [14].

Da Prato and Lunardi [11] studied equation (1.1) (see also Da PratoLunardi [12], Clément-Da Prato [9]) under several conditions on $A$ and $a(\cdot)$. In particular, among other hypotheses, they assume that $A$ generates an analytic semigroup. We note, however, that these authors also consider more general operator valued kernels and non periodic problems.

In this work we rely on the recent papers by Arendt-Bu [3], Arendt-Bu [5], Arendt-Batty-Bu [4] where the above problem is studied with $a(\cdot) \equiv 0$. In these papers, the authors establish operator-valued Fourier multiplier theorems and apply them to study maximal regularity of the classical abstract non homogeneous Cauchy problem

$$
\left\{\begin{array}{l}
u^{\prime}(t)=A u(t)+f(t), 0 \leq t \leq 2 \pi \\
u(0)=u(2 \pi)
\end{array}\right.
$$

One remarkable fact is that in the context of resolvents of closed linear operators in Banach spaces, $L^{p}$-multipliers can be completely characterized. See for example [3, Theorem 2.3] and [5, Theorem 4.1] for a precise formulation 
of this fact. This is in contrast to the general situation where, even in the scalar case, a complete characterization exists only for $p=1$ and $p=2$.

Our case is more difficult to handle due to the complicated structure of the resolvent. We are able to establish maximal regularity in all the above spaces: Lebesgue-Bochner, Hölder and Besov spaces. In the case of Hölder spaces, which was considered by Da Prato-Lunardi [11], we obtain a complete and very simple characterization of maximal regularity only in terms of boundedness of $\left\{b_{k}\left(b_{k}-A\right)^{-1}\right\}_{k \in \mathbb{Z}}$ where $b_{k}=\frac{i k}{1+\tilde{a}(i k)}$ and $\tilde{a}(\lambda)$ denotes the Laplace transform of $a(\cdot)$. The conditions that we impose on the kernel $a(\cdot)$ are satisfied by a large class of functions. For example, the Analytic Representation Theorem ([2, Theorem 2.6.1 ]) gives a characterization of a subclass of functions which fit into our framework. Moreover, the important example $a(t)=b e^{-c t}$ with $c>0$ and $b \in \mathbb{R}$ is shown to satisfy our assumptions. For this and more examples, see the monograph [16] by J. Prüss, especially Chapter II, section 9 and Chapter III, section 13.

We remark that in the case where $a \equiv 0$, which corresponds to problem (1.3), we recover the results of [3, Theorem 2.3], [5, Theorem 4.1] and in particular [4, Theorem 4.2].

Compared with the papers [11] and [12], our assumptions are weaker. We do not make any parabolicity assumption on the operator $A$, not even that $A$ generates a semigroup.

In the theory of Volterra integral equations in Banach spaces, the notion of $k$-regular kernels plays a fundamental rôle (see e.g. Prüss [16, Theorem 3.1, p.73] and [16, Definition 3.3, p.69]). In our analysis, we introduce a 
discrete version of this concept (see Definition 3) and use it to obtain a characterization of multipliers in terms of boundedness of the resolvent.

The present paper is organized as follows: In section 2, we consider wellposedness of $(1.1)$ in the Lebesgue Bochner spaces $L_{2 \pi}^{p}(\mathbb{R} ; X)$. Here the main result involves $U M D$-spaces and R-boundedness. The third section is devoted to maximal regularity in $C^{\alpha}(0,2 \pi ; X)$ and $B_{p q}^{s}(0,2 \pi ; X)$. In each case, the appropriate notion of strong solution is defined.

\section{Maximal Regularity on $L^{p}(0,2 \pi ; X): R$-Boundedness.}

For a function $f \in L^{1}(0,2 \pi ; X)$, we denote by $\hat{f}(k), k \in \mathbb{Z}$ the k-th Fourier coefficient of $f$ :

$$
\hat{f}(k)=\frac{1}{2 \pi} \int_{0}^{2 \pi} e_{-k}(t) f(t) d t
$$

where $e_{k}(t)=e^{i k t}, t \in \mathbb{R}$. Given $a \in L_{l o c}^{1}\left(\mathbb{R}_{+}\right)$and $u:[0,2 \pi] \rightarrow X$ ( extended by periodicity to $\mathbb{R}$ ) we obtain, under appropriate assumptions on $a(t)$, that for $F(t)=\int_{-\infty}^{t} a(t-s) u(s) d s$,

$$
\hat{F}(k)=\tilde{a}(i k) \hat{u}(k), k \in \mathbb{Z}
$$

where $\tilde{a}(\lambda)=\int_{0}^{\infty} e^{-\lambda t} a(t) d t$ denotes the Laplace transform of $f$. We shall return to this point later.

Let $X, Y$ be Banach spaces. We denote by $\mathcal{L}(X, Y)$ the set of all bounded linear operators from $X$ to $Y$. When $X=Y$, we write simply $\mathcal{L}(X)$. For a linear operator $A$ on $X$, we denote its resolvent set by $\rho(A)$.

We begin with some preliminaries about operator-valued Fourier multipliers. 
Definition 2.1. For $1 \leq p \leq \infty$, we say that a sequence $\left\{M_{k}\right\}_{k \in \mathbb{Z}} \subset$ $\mathcal{L}(X, Y)$ is an $L^{p}$-multiplier, if for each $f \in L^{p}(0,2 \pi ; X)$ there exists $u \in$ $L^{p}(0,2 \pi ; Y)$ such that

$$
\hat{u}(k)=M_{k} \hat{f}(k) \text { for all } k \in \mathbb{Z} \text {. }
$$

It follows from the uniqueness theorem of Fourier series that $u$ is uniquely determined by $f$.

For $j \in \mathbb{N}$, denote by $r_{j}$ the $j$-th Rademacher function on $[0,1]$, i.e. $r_{j}(t)=$ $\operatorname{sgn}\left(\sin \left(2^{j} \pi t\right)\right)$. For $x \in X$ we denote by $r_{j} \otimes x$ the vector valued function $t \rightarrow r_{j}(t) x$

Definition 2.2. A family $\mathbf{T} \subset \mathcal{L}(X, Y)$ is called $R$-bounded if there exists $c_{q} \geq 0$ such that

$$
\left\|\sum_{j=1}^{n} r_{j} \otimes T_{j} x_{j}\right\|_{L^{q}(0,1 ; X)} \leq c_{q}\left\|\sum_{j=1}^{n} r_{j} \otimes x_{j}\right\|_{L^{q}(0,1 ; X)}
$$

for all $T_{1}, \ldots, T_{n} \in \mathbf{T}, x_{1}, \ldots, x_{n} \in X$ and $n \in \mathbb{N}$, where $1 \leq q<\infty$. We denote by $R_{q}(\mathbf{T})$ the smallest constant $c_{q}$ such that (2.2) holds.

The concept of $R$-boundedness was introduced by Bourgain [6]. It plays a fundamental role in recent work by Clément-de Pagter-Sukochev-Witvliet [10], Weis [18, 19], Clément- Prüss [9] and Arendt-Bu [3]. It is clear from the definition that any $R$-bounded family is bounded. The converse of this assertion holds only in spaces which are isomorphic to Hilbert spaces. For more details, we refer to Arendt-Bu $[3,4]$. 
a) Let $\mathbf{S}, \mathbf{T} \subset \mathcal{L}(X)$ be $R$-bounded sets, then $\mathbf{S} \cdot \mathbf{T}:=\{S \cdot T: S \in \mathbf{S}, T \in$ $\mathbf{T}\}$ is $R$ - bounded and

$$
R_{p}(\mathbf{S} \cdot \mathbf{T}) \leq R_{p}(\mathbf{S}) \cdot R_{p}(\mathbf{T})
$$

b) Also, each subset $M \subset \mathcal{L}(X)$ of the form $M=\{\lambda I: \lambda \in \Omega\}$ is $R$ bounded whenever $\Omega \subset \mathbb{C}$ is bounded ( $I$ denotes the identity operator on $X)$. This follows from Kahane's inequality (see [3, Lemma 1.7]). We shall use this remark frequently.

The following theorem, due to Arendt-Bu [3, Theorem 1.3], is the discrete analogue of the operator-valued version of Mikhlin's theorem. It is concerned with $U M D$ spaces. Examples of $U M D$ spaces include Hilbert spaces, $L^{p}(\Omega, \mu), 1<p<\infty, L^{p}(\Omega, \mu ; X), 1<p<\infty$ when $X$ is a $U M D$ space and the Schatten-von Neumann classes $C_{p}(H), 1<p<\infty$ of operators on a Hilbert space. Every $U M D$ space is superreflexive. The spaces $L^{1}(\Omega, \mu), L^{\infty}(\Omega, \mu)$ (if infinite dimensional) and $C^{\alpha}([0,2 \pi] ; X)$ are not reflexive and therefore not $U M D$. More information on $U M D$ spaces can be found in Amann [1], Bourgain [6], De Pagter-Witvliet [13] and Prüss [16].

Theorem 2.4. (Operator-valued Marcinkiewicz multiplier theorem)

Let $X, Y$ be $U M D$-spaces and $M_{k} \in \mathcal{L}(X, Y)(k \in \mathbb{Z})$. If the sets $\left\{M_{k}\right\}_{k \in \mathbb{Z}}$ and $\left\{k\left(M_{k+1}-M_{k}\right)\right\}_{k \in \mathbb{Z}}$ are $R$-bounded, then $\left\{M_{k}\right\}_{k \in \mathbb{Z}}$ is an $L^{p}$-multiplier for $1<p<\infty$. 
If $X=Y$ is a $U M D$ space and $M_{k}=m_{k} I$ with $m_{k} \in \mathbb{C}$, then the condition

$$
\sup _{k}\left|m_{k}\right|+\sup _{k}\left|k\left(m_{k+1}-m_{k}\right)\right|<\infty
$$

implies that the set $\left\{M_{k}\right\}_{k \in \mathbb{Z}}$ is an $L^{p}$-multiplier. (see [3] or [1, Theorem 4.4.3]).

The following concept of k-regularity $(k=1,2)$ is the discrete analog for the notion of k-regularity introduced by Prüss [16, Chapter I, Section 3.2].

Definition 2.6. A sequence $\left\{a_{k}\right\}_{k \in \mathbb{Z}} \subset \mathbb{C} \backslash\{0\}$ is called

a) 1-regular if the sequence $\left\{\frac{k\left(a_{k+1}-a_{k}\right)}{a_{k}}\right\}_{k \in \mathbb{Z}}$ is bounded.

b) 2-regular if it is 1-regular and the sequence $\left\{\frac{k^{2}\left(a_{k+1}-2 a_{k}+a_{k-1}\right)}{a_{k}}\right\}_{k \in \mathbb{Z}}$ is bounded.

Example 2.7.

It is not difficult to see that the sequence $a_{k}=\frac{b}{i k+c}$, where $b \in \mathbb{R}$ and $c>0$, is 2-regular.

Proposition 2.8. Let $A$ be a closed linear operator defined on the Banach space $X$. Let $\left\{a_{k}\right\}_{k \in \mathbb{Z}} \in \mathbb{C} \backslash\{0\}$ be a 1-regular sequence such that $\left\{\frac{1}{a_{k}}\right\}_{k \in \mathbb{Z}} \subset$ $\rho(A)$. Then the following assertions are equivalent

(i) $\left\{\left(I-a_{k} A\right)^{-1}\right\}_{k \in \mathbb{Z}}$ is an $L^{p}$-multiplier, $1<p<\infty$.

(ii) $\left\{\left(I-a_{k} A\right)^{-1}\right\}_{k \in \mathbb{Z}}$ is R-bounded.

Proof. By [3, Proposition 1.11] it follows that (i) implies (ii). Conversely, define $M_{k}=\left(I-a_{k} A\right)^{-1}$. By Theorem 2.4 is sufficient to prove that the set 
$\left\{k\left(M_{k+1}-M_{k}\right)\right\}_{k \in \mathbb{Z}} \subset \mathcal{L}(X)$ is $R$-bounded. In fact,

$$
\begin{aligned}
k\left(M_{k+1}-M_{k}\right) & =k\left[\left(I-a_{k+1} A\right)^{-1}-\left(I-a_{k} A\right)^{-1}\right] \\
& =k\left(I-a_{k+1} A\right)^{-1}\left[\left(I-a_{k} A\right)-\left(I-a_{k+1} A\right)\right]\left(I-a_{k} A\right)^{-1} \\
& =k\left(I-a_{k+1} A\right)^{-1}\left[a_{k+1}-a_{k}\right] A\left(I-a_{k} A\right)^{-1} \\
& =k\left(a_{k+1}-a_{k}\right)\left(I-a_{k+1} A\right)^{-1} \frac{1}{a_{k}}\left[\left(I-a_{k} A\right)^{-1}-I\right] \\
& =\frac{k\left(a_{k+1}-a_{k}\right)}{a_{k}}\left(I-a_{k+1} A\right)^{-1}\left[\left(I-a_{k} A\right)^{-1}-I\right]
\end{aligned}
$$

hence the result follows from Remark 2.3.

Let $a \in L_{l o c}^{1}\left(\mathbb{R}_{+}\right)$and suppose that $\tilde{a}(i k)$ exists for all $k \in \mathbb{Z}$. We assume that $\lambda \rightarrow \tilde{a}(\lambda)$ admits an extension to a sector containing the imaginary axis, and still denote this extension by $\tilde{a}$. For example we may assume that $a$ is of subexponential growth and 1-regular (in the sense on [16]). For the properties of Laplace transforms of such functions we refer to Prüss [16, Chapter 2, Lemma 8.1]. Another class of interest is the one characterized in [2, Theorem 2.6.1], but our results apply to a more general class as Example 2.16 (1) below shows. Other interesting conditions are considered in Da Prato-Lunardi [12, Section 5]. We adopt throughout the following notations:

$$
\begin{aligned}
c_{k} & =\tilde{a}(i k) ; \\
b_{k} & =\frac{1}{a_{k}}=\frac{i k}{1+\tilde{a}(i k)} \text { for all } k \in \mathbb{Z} \backslash\{0\} ; \quad b_{0}=0,
\end{aligned}
$$

and $R(\lambda, A)=(\lambda-A)^{-1}$ whenever $\lambda \in \rho(A)$. 
As announced in the introduction, we shall frequently identify the spaces of (vector or operator-valued) functions defined on $[0,2 \pi]$ to their periodic extensions to $\mathbb{R}$. Thus, in this section, we consider the space $L_{2 \pi}^{p}(\mathbb{R} ; X)$ (denoted also $\left.L^{p}(0,2 \pi ; X), 1 \leq p \leq \infty\right)$ of all $2 \pi$-periodic Bochner measurable $X$-valued functions $f$ such that the restriction of $f$ to $[0,2 \pi]$ is $p$-integrable.

The following hypothesis will be fundamental for our purposes.

(H1) $\left\{c_{k}\right\}_{k \in \mathbb{Z}},\left\{k\left(c_{k+1}-c_{k}\right)\right\}_{k \in \mathbb{Z}}$ and $\left\{\frac{1}{c_{k}+1}\right\}_{k \in \mathbb{Z}}$ are bounded sequences.

Note that $(H 1)$ implies that $\left\{b_{k}\right\}$ is 1-regular. This follows from the identity

$$
k \frac{\left(b_{k+1}-b_{k}\right)}{b_{k}}=\frac{1}{1+c_{k+1}}\left[\left(1+c_{k}\right)-k\left(c_{k+1}-c_{k}\right)\right]
$$

Lemma 2.9. Let $X$ be a UMD space. Under assumption (H1), the sequences $\left\{\left(1+c_{k}\right) I\right\}_{k \in \mathbb{Z}},\left\{\frac{\left(1+c_{k}\right)}{i k} I\right\}_{k \in \mathbb{Z} \backslash\{0\}}$ and $\left\{\left(\frac{1}{1+c_{k}}\right) I\right\}_{k \in \mathbb{Z}}$ are $L^{p}$-multipliers.

Proof. We have

$$
k\left(\frac{1}{1+c_{k+1}}-\frac{1}{1+c_{k}}\right)=k \frac{\left(c_{k}-c_{k+1}\right)}{\left(1+c_{k+1}\right)\left(1+c_{k}\right)}
$$

and

$$
i k\left(a_{k+1}-a_{k}\right)=\frac{1}{k+1}\left[k\left(c_{k+1}-c_{k}\right)-\left(1+c_{k}\right)\right]
$$

Hence the result follows from Remark 2.5. 
In what follows, we denote by $H_{p e r}^{a, p}$ the space of all $u \in L^{p}(0,2 \pi ; X)$ for which there exists $v \in L^{p}(0,2 \pi ; X)$ such that $\hat{v}(k)=\frac{i k}{1+\tilde{a}(i k)} \hat{u}(k)$ for all $k \in \mathbb{Z}$. If $a \equiv 0$ we denote $H_{\text {per }}^{1, p}:=H_{\text {per }}^{0, p}$ (cf. [3, Section 2] ).

Remark 2.10.

Under assumption $(H 1)$ we have, by Lemma 2.9 , that $1+c_{k}$ and $\frac{1}{1+c_{k}}$ are $L^{p}$ - multipliers and hence $H_{p e r}^{1, p}=H_{p e r}^{a, p}$.

Definition 2.11. A function $u \in H_{\text {per }}^{a, p}$ is called a strong $L^{p}$ - solution of (1.1) if $u(t) \in D(A)$ and equation (1.1) holds for almost all $t \in[0,2 \pi]$.

The following is the main result of this section. Recall that $b_{k}=\frac{i k}{1+c_{k}}$, where $c_{k}=\tilde{a}(i k)$.

Theorem 2.12. Let $X$ be a $U M D$ space and $A: D(A) \subset X \rightarrow X$ be a closed linear operator. Assume that the sequence $\left\{c_{k}\right\}_{k \in \mathbb{Z}}$ satisfies $(H 1)$. Then the following assertions are equivalent for $1<p<\infty$ :

(i) For every $f \in L_{2 \pi}^{p}(\mathbb{R} ; X)$ there exists a unique strong $L^{p}$ - solution of (1.1).

(ii) $\left\{b_{k}\right\}_{k \in \mathbb{Z}} \subseteq \rho(A)$ and $\left.\left\{b_{k}\left(b_{k} I-A\right)^{-1}\right)\right\}_{k \in \mathbb{Z}}$ is an $L^{p}$-multiplier.

(iii) $\left\{b_{k}\right\}_{k \in \mathbb{Z}} \subseteq \rho(A)$ and $\left\{b_{k}\left(b_{k} I-A\right)^{-1}\right\}_{k \in \mathbb{Z}}$ is $R$-bounded.

Proof. $(i) \Longrightarrow(i i)$. Follows the same lines as the proof of $[3$, Theorem 2.3] . The key point is to take advantage of the equation (2.1) and Lemma 2.9. Let $k \in \mathbb{Z}$ and $y \in X$. Define $f=e_{k} \otimes y$. There exists $u \in H_{\text {per }}^{a, p}$ such that $u^{\prime}(t)=A u(t)+\int_{-\infty}^{t} a(t-s) A u(s) d s+f(t)$. Taking Fourier transforms on both sides we obtain that $\hat{u}(k) \in D(A)$ and $i k \hat{u}(k)=A \hat{u}(k)+\tilde{a}(i k) A \hat{u}(k)+\hat{f}(k)=$ 
$A \hat{u}(k)+\tilde{a}(i k) \hat{u}(k)+y$. Thus, $(i k I-(1+\tilde{a}(i k)) A)$ is surjective. Let $x \in D(A)$. If $(i k I-(1+\tilde{a}(i k)) A) x=0$, that is $A x=\frac{i k}{1+\tilde{a}(i k)} x=b_{k} x$ then $u(t)=e^{i k t} x$ defines a periodic solution of $u^{\prime}(t)=A u(t)+\int_{-\infty}^{t} a(t-s) A u(s) d s=0$. In fact,

$$
\begin{aligned}
A u(t)+\int_{-\infty}^{t} a(t-s) A u(s) d s & =e^{i k t} A x+\int_{-\infty}^{t} a(t-s) e^{i k s} A x d s \\
& =e^{i k t} A x+\int_{0}^{\infty} a(s) e^{i k(t-s)} A x d s \\
& =e^{i k t} A x+e^{i k t} \int_{0}^{\infty} a(s) e^{-i k s} d s A x \\
& =e^{i k t} A x+e^{i k t} \tilde{a}(i k) A x \\
& =e^{i k t}(1+\tilde{a}(i k)) A x \\
& =i k e^{i k t} x=u^{\prime}(t) .
\end{aligned}
$$

Hence $u=0$ by the assumption of uniqueness and thus $x=0$. Since $A$ is closed, we conclude that $\left\{b_{k}\right\}_{k \in \mathbb{Z}} \subset \rho(A)$.

Next we show that $\left\{\left(I-a_{k} A\right)^{-1}\right\}_{k \in \mathbb{Z}}$ is an $L^{p}$ - multiplier. Let $f \in$ $L^{p}(0,2 \pi ; X)$. By hypothesis, there exists a unique $u \in H_{\text {per }}^{a, p}$ such that $u^{\prime}(t)=A u(t)+\int_{-\infty}^{t} a(t-s) A u(s) d s+f(t)$. Taking Fourier transforms, we deduce that $\hat{u}(k) \in D(A)$ and $(i k I-(1+\tilde{a}(i k)) A) \hat{u}(k)=\hat{f}(k)$ or $i k a_{k}\left(\frac{1}{a_{k}} I-A\right) \hat{u}(k)=\hat{f}(k)$ for all $k \in \mathbb{Z}$. Hence,

$$
i k \hat{u}(k)=\left(I-a_{k} A\right)^{-1} \hat{f}(k) \text { for all } k \in \mathbb{Z} .
$$

Note that by definition of $H_{p e r}^{a, p}$ there exists $v \in L^{p}(0,2 \pi ; X)$ such that $(1+\tilde{a}(i k)) \hat{v}(i k)=i k \hat{u}(k)=\left(I-a_{k} A\right)^{-1} \hat{f}(k)$ for all $k \in \mathbb{Z}$. Hence, to finish the proof it is sufficient to prove that the set $\{1+\tilde{a}(i k)\}_{k \in \mathbb{Z}}=\left\{1+c_{k}\right\}_{k \in \mathbb{Z}}$ 
satisfies Mikhlin's conditions (cf. Remark 2.5 ), but this is contained in Lemma 2.9.

$(i i) \Longrightarrow(i)$. Let $f \in L^{p}(0,2 \pi ; X)$. By Lemma 2.9 , the sequence $\left\{\frac{1}{1+c_{k}} I\right\}$ is an $L^{p}$ - multiplier. Hence, there exists $g \in L^{p}(0,2 \pi ; X)$ such that

$$
\hat{g}(k)=\frac{1}{1+c_{k}} \hat{f}(k)
$$

By uniqueness of Fourier coefficients, we obtain $f(t)=g(t)+\int_{-\infty}^{t} a(t-$ $s) g(s) d s$.

Since $g \in L^{p}(0,2 \pi ; X)$ we use the hypothesis, to get a function $v \in$ $L^{p}(0,2 \pi ; X)$ such that $\hat{v}(k)=\left(I-a_{k} A\right)^{-1} \hat{g}(k)=b_{k}\left(b_{k} I-A\right)^{-1} \hat{g}(k)$. Hence $a_{k} \hat{v}(k)=\left(b_{k} I-A\right)^{-1} \hat{g}(k)$.

Because the sequence $\left\{a_{k} I\right\}_{k \in \mathbb{Z}}$ is an $L^{p}$-multiplier by Lemma 2.9, we conclude that there exists $u \in L^{p}(0,2 \pi ; X)$ such that

$$
\hat{u}(k)=a_{k} \hat{v}(k)=\left(b_{k} I-A\right)^{-1} \hat{g}(k) .
$$

In particular, $u \in H_{\text {per }}^{a, p}$.

Now, for all $x \in D(A)$, the identity $\left(b_{k} I-A\right)^{-1} A x=b_{k}\left(b_{k} I-A\right)^{-1} x-x$ shows that $\left(b_{k} I-A\right)^{-1}$ is an $\mathcal{L}(X ; D(A))$ - multiplier. Hence $u \in L^{p}(0,2 \pi ; D(A))$. Finally, we have $b_{k} \hat{u}(k)-A \hat{u}(k)=b_{k}\left(b_{k} I-A\right)^{-1} \hat{g}(k)-A\left(b_{k} I-A\right)^{-1} \hat{g}(k)=$ $\hat{g}(k)$ or $\frac{i k}{1+\tilde{a}(i k)} \hat{u}(k)-A \hat{u}(k)=\hat{g}(k)$. Hence $i k \hat{u}(k)=(1+\tilde{a}(i k)) A \hat{u}(k)+(1+\tilde{a}(i k)) \hat{g}(k)=A \hat{u}(k)+\tilde{a}(i k) A \hat{u}(k)+\hat{f}(k)$. It follows from the uniqueness theorem of Fourier coefficients that (1.1) holds for almost all $t \in[0,2 \pi]$. Since by Lemma 2.9 , the sequence $\left\{\left(1+c_{k}\right) I\right\}$ is an $L^{p}$-multiplier, there exists $w \in L^{p}(0,2 \pi ; X)$ such that $\hat{w}(k)=\left(1+c_{k}\right) \hat{v}(k)=$ 
$i k \hat{u}(k)$. Hence by [3, Lemma 2.1], it follows that $u(0)=u(2 \pi)$. We have proved that $u$ is a strong $L^{p}$ - solution of (1.1). It remains to show uniqueness.

Let $u \in H_{\text {per }}^{a, p} \cap L^{p}(0,2 \pi ; D(A))$ be such that

$$
u^{\prime}(t)-A u(t)-\int_{-\infty}^{t} a(t-s) A u(s) d s=0
$$

then $\hat{u}(k) \in D(A)$ and $(i k I-(1+\tilde{a}(i k)) A) \hat{u}(k)=0$. Since $\frac{i k}{1+\tilde{a}(i k)} \in \rho(A)$ this implies that $\hat{u}(k)=0$ for all $k \in \mathbb{Z}$ and thus $u=0$.

$(i i) \Leftrightarrow(i i i)$. Follows from Proposition 2.8 and the observation that $\left\{b_{k}\right\}$ is 1-regular if and only if $\left\{a_{k}\right\}$ is 1-regular. In fact, we can write

$$
k \frac{\left(b_{k+1}-b_{k}\right)}{b_{k}}=-k \frac{\left(a_{k+1}-a_{k}\right)}{a_{k}} \cdot \frac{a_{k}}{a_{k+1}}
$$

and note that $\left|k\left(\frac{a_{k+1}}{a_{k}}-1\right)\right| \leq M$ for some $M>0$, implies $\frac{a_{k+1}}{a_{k}} \rightarrow 1$ and $\frac{a_{k}}{a_{k+1}} \rightarrow 1$. Hence $\left\{k \frac{\left(b_{k+1}-b_{k}\right)}{b_{k}}\right\}$ is bounded.

Corollary 2.13. Let $H$ be a Hilbert space and $A: D(A) \subset H \rightarrow H$ be a closed linear operator. If the sequence $\left\{c_{k}\right\}_{k \in \mathbb{Z}}$ satisfies (H1) then, for $1<p<\infty$, the following assertions are equivalent:

(i) For every $f \in L_{\text {per }}^{p}(\mathbb{R} ; H)$ there exists a unique strong $L^{p}$ - solution of (1.1).

(ii) $\left\{b_{k}\right\}_{k \in \mathbb{Z}} \subset \rho(A)$ and $\sup _{k}\left\|b_{k}\left(b_{k} I-A\right)^{-1}\right\|<\infty$.

Proof. This follows from Theorem 2.12, Proposition 2.8 and the fact that in the context of Hilbert spaces, $R$-boundedness and boundedness are identical concepts. This in turn follows from Plancherel's theorem and the fact 
that the Rademacher system $\left\{r_{j}(t)\right\}$ is an orthonormal family in $L^{2}(0,1 ; \mathbb{C})$ (see Clément-de Pagter-Sukochev-Witvliet [10]).

The solution $u(\cdot)$ given by Theorem 2.12 actually satisfies the following maximal regularity property. We will adopt the notation:

$$
a \dot{*} A u=\int_{-\infty}^{\cdot} a(\cdot-s) A u(s) d s
$$

Corollary 2.14. In the context of Theorem 2.12, if condition (ii) is valid we have: $u^{\prime}, A u, a \dot{*} A u \in L^{p}(0,2 \pi ; X)$. Moreover there exists a constant $C>0$ independent of $f \in L^{p}(0,2 \pi ; X)$ such that

$$
\left\|u^{\prime}\right\|_{L^{p}(0,2 \pi ; X)}+\|A u\|_{L^{p}(0,2 \pi ; X)}+\|a \dot{*} A u\|_{L^{p}(0,2 \pi ; X)} \leq C\|f\|_{L^{p}(0,2 \pi ; X)}
$$

Proof. The first statement follows from the proof of Theorem 2.12. We verify this for $A u$ : From $(2.7)$ we have $\hat{u}(k)=\left(b_{k} I-A\right)^{-1} \hat{g}(k)$ hence

$$
A \hat{u}(k)=b_{k}\left(b_{k} I-A\right)^{-1} \hat{g}(k)-\hat{g}(k) .
$$

Since $g \in L^{p}(0,2 \pi ; X)$ and $\left(I-a_{k} A\right)^{-1}=b_{k}\left(b_{k} I-A\right)^{-1}$ is an $L^{p}$-multiplier, the claim follows. On the other hand, $\int_{-\infty}^{t} a(t-s) A u(s) d s=u^{\prime}(t)-A u(t)-$ $f(t)$, which implies $\int_{-\infty}^{\cdot} a(\cdot-s) A u(s) d s \in L^{p}(0,2 \pi ; X)$. The second statement is a consequence of the Closed Graph Theorem.

Remark 2.15.

Fejer's Theorem (see [3, Proposition 1.1] or [2, Theorem 4.2.19]) can be used to construct the solution $u(\cdot)$ given by Theorem 2.12. More precisely, 
we have:

$$
u(\cdot)=\lim _{n \rightarrow \infty} \frac{1}{n+1} \sum_{m=0}^{n} \sum_{k=-m}^{m} e_{k} \otimes R\left(b_{k}, A\right)(1+\tilde{a}(i k))^{-1} \hat{f}(k)
$$

with convergence in $L^{p}(0,2 \pi ; X)$.

Example 2.16.

(1) Suppose $T>0$ is given and $a(t)=\chi_{[0, T]}(t)$ where $\chi_{[0, T]}$ denotes the characteristic function of $[0, T]$. Then (1.1) takes the particularly simple form:

$$
u^{\prime}(t)=A u(t)+\int_{0}^{T} A u(t-s) d s+f(t), \quad 0 \leq t \leq 2 \pi
$$

In this case, $\tilde{a}(\lambda)=\frac{1-e^{-\lambda T}}{\lambda}, \lambda \neq 0$. We set $\tilde{a}(0)=T$. Therefore $c_{k}=$ $\tilde{a}(i k)=\frac{1-e^{-i k T}}{i k}=\frac{2}{k} e^{-i k T / 2} \sin \frac{k T}{2}$ for $k \in \mathbb{Z} \backslash\{0\}$. Multiplier conditions on $\left(1+c_{k}\right),\left(\frac{1+c_{k}}{i k}\right)$ are readily verified. As for $d_{k}:=\frac{1}{1+c_{k}}$, we have $d_{k}=$ $\frac{i k}{1+i k-e^{-i k T}}$ which is bounded. Finally

$$
\begin{aligned}
d_{k+1}-d_{k} & =\frac{i k\left(e^{-i T}-1\right) e^{-i k T}+i\left(1-e^{-i k T}\right)}{\left(1+i(k+1)-e^{-i(k+1) T}\right)\left(1+i k-e^{-i k T}\right)} \\
& =\frac{i e^{-i k T}\left(-1-k+e^{i T}\right)+i}{\left(1+i(k+1)-e^{-i(k+1) T}\right)\left(1+i k-e^{-i k T}\right)}
\end{aligned}
$$

so that $k\left(d_{k+1}-d_{k}\right)$ is bounded. Therefore, $(H 1)$ is satisfied.

(2) Let $a(t)=b e^{-c t},(c>0)$. We will make the further assumption that $b+c \neq 0$. Here we have $\tilde{a}(\lambda)=\frac{b}{\lambda+c}, \operatorname{Re} \lambda>c$, hence $c_{k}=\frac{b}{i k+c}, k \in \mathbb{Z}$. Clearly $\left\{c_{k}\right\}$ is bounded and $\left\{k c_{k}\right\}$ is bounded as well. It remains to examine the multiplier conditions on $\frac{1}{1+c_{k}}$. But $1+c_{k}=\frac{b+c+i k}{c+i k}$ implies $d_{k}:=\frac{1}{1+c_{k}}=1-\frac{b}{b+c+i k}$ which is bounded. The boundedness of $k\left(d_{k+1}-d_{k}\right)$ is easily verified, thus $(H 1)$ is also satisfied in this case. This example was 
considered by Da Prato and Lunardi [11] and is very important in the theory of linear Volterra equations which treat practical applications. For this and more examples, see J. Prüss [16, Chapter I, section 5, p.131].

\section{Maximal Regularity on Hölder and Besov spaces:}

\section{M-BOUNDEDNESS}

In the paper [5], Arendt and Bu showed that the analogue of Marcinkiewicz's operator-valued Fourier multiplier theorem on $L^{p}$ holds for the Besov space $B_{p, q}^{s}(\mathbb{T} ; X)$ if and only if $1<p<\infty$ and $X$ is an $U M D$ - space. Here, $\mathbb{T}$ denotes the one dimensional torus $\mathbb{R} / \mathbb{Z}$ which we often identify with $[0,2 \pi]$. Introducing stronger conditions these authors obtained a periodic (operatorvalued) Fourier multiplier theorem which is valid without restrictions on the indices or the space. In this section, we use the results of [5] to characterize maximal regularity of equation (1.1). We briefly recall the definition of periodic Besov spaces in the vector valued case introduced in [5]. For the scalar case, see Triebel [17, Chapter 9]. An approach to periodic Besov spaces based on semigroup theory and abstract interpolation is presented in [7, Chapter 4].

Let $\mathcal{S}$ be the Schwartz space on $\mathbb{R}$ and let $\mathcal{S}^{\prime}$ be the space of all tempered distributions on $\mathbb{R}$. Let $\Phi(\mathbb{R})$ be the set of all systems $\phi=\left\{\phi_{j}\right\}_{j \geq 0} \subset \mathcal{S}$ satisfying

$$
\begin{gathered}
\operatorname{supp}\left(\phi_{0}\right) \subset[-2,2] \\
\operatorname{supp}\left(\phi_{j}\right) \subset\left[-2^{j+1},-2^{j-1}\right] \cup\left[2^{j-1}, 2^{j+1}\right], \quad j \geq 1
\end{gathered}
$$




$$
\sum_{j \geq 0} \phi_{j}(t)=1, \quad t \in \mathbb{R}
$$

and for $\alpha \in \mathbb{N} \cup\{0\}$, there exists $C_{\alpha}>0$ such that

$$
\sup _{j \geq 0, x \in \mathbb{R}} 2^{\alpha j}\left\|\phi_{j}^{(\alpha)}(x)\right\| \leq C_{\alpha}
$$

Let $1 \leq p, q \leq \infty, s \in \mathbb{R}$ and $\phi=\left(\phi_{j}\right)_{j \geq 0} \in \Phi(\mathbb{R})$. The $X$ - valued periodic Besov spaces are defined by

$$
B_{p, q}^{s, \phi}=\left\{f \in \mathcal{D}^{\prime}(\mathbb{T} ; X):\|f\|_{B_{p, q}^{s, \phi}}=\left(\sum_{j \geq 0} 2^{s j q}\left\|\sum_{k \in \mathbb{Z}} e_{k} \otimes \phi_{j}(k) \hat{f}(k)\right\|_{p}^{q}\right)^{1 / q}<\infty\right\}
$$

The space $B_{p, q}^{s, \phi}$ is independent of $\phi \in \Phi(\mathbb{R})$ and the norms $\|\cdot\|_{B_{p, q}^{s, \phi}}$ are equivalent. We will simply denote $\|\cdot\|_{B_{p, q}^{s, \phi}}$ by $\|\cdot\|_{B_{p, q}^{s}}$ for some $\phi \in \Phi(\mathbb{R})$. See [5, section 1] for more details.

Definition 3.1. Let $X$ and $Y$ Banach spaces and let $\left\{M_{k}\right\}_{k \in \mathbb{Z}} \subset \mathcal{L}(X, Y)$. We will say that $\left\{M_{k}\right\}_{k \in \mathbb{Z}}$ is an $B_{p, q}^{s}$-multiplier, if for each $f \in B_{p, q}^{s}(\mathbb{T} ; X)$ there exists $u \in B_{p, q}^{s}(\mathbb{T} ; Y)$ such that

$$
\hat{u}(k)=M_{k} \hat{f}(k) \text { for all } k \in \mathbb{Z} .
$$

The following condition on sequences $\left\{M_{k}\right\}_{k \in \mathbb{Z}} \subset \mathcal{L}(X, Y)$ was introduced in [4] to study Fourier multipliers for Hölder continuous functions. It is also used in the study of multipliers of Besov spaces of which the spaces $C^{\alpha}(\mathbb{T} ; X)$ of $X$-valued Hölder continuous functions are a special instance.

Definition 3.2. We say that a sequence $\left\{M_{k}\right\}_{k \in \mathbb{Z}} \subset \mathcal{L}(X, Y)$ is $M$ - bounded if

$$
\sup _{k \in \mathbb{Z}}\left\|M_{k}\right\|<\infty, \quad \sup _{k \in \mathbb{Z}}\left\|k\left(M_{k+1}-M_{k}\right)\right\|<\infty
$$




$$
\sup _{k \in \mathbb{Z}}\left\|k^{2}\left(M_{k+1}-2 M_{k}+M_{k-1}\right)\right\|<\infty .
$$

The following general multiplier theorem is due to Arendt-Bu [5, Theorem $3.5]$.

Theorem 3.3. Let $X$ and $Y$ Banach spaces and let $\left\{M_{k}\right\}_{k \in \mathbb{Z}} \subset \mathcal{L}(X, Y)$ be a $M$ - bounded sequence. Then for $1 \leq p, q \leq \infty, s \in \mathbb{R},\left\{M_{k}\right\}_{k \in \mathbb{Z}}$ is a $B_{p, q}^{s}$-multiplier.

We are now in a position to prove the following proposition which is the analogue of Proposition 2.8.

Proposition 3.4. Let $A$ be a closed linear operator defined on the Banach space $X$. Let $\left\{b_{k}\right\}_{k \in \mathbb{Z}} \in \mathbb{C} \backslash\{0\}$ be a 2-regular sequence such that $\left\{b_{k}=\right.$ $\left.\frac{1}{a_{k}}\right\}_{k \in \mathbb{Z}} \subset \rho(A)$. Let $M_{k}=\left(I-a_{k} A\right)^{-1}$. Then the following assertions are equivalent

(i) $\left\{\left(I-a_{k} A\right)^{-1}\right\}_{k \in \mathbb{Z}}$ is a $B_{p, q}^{s}$-multiplier, $1 \leq p \leq \infty, s \in \mathbb{R}$,

(ii) $\left\{\left(I-a_{k} A\right)^{-1}\right\}_{k \in \mathbb{Z}}$ is bounded.

Proof. $\quad(i) \Rightarrow(i i)$. It follows from the Closed Graph Theorem that there exists $C>0$ such that for $f \in B_{p, q}^{s}(\mathbb{T} ; X)$, we have

$$
\left\|\sum_{k \in \mathbb{Z}} e_{k} \otimes M_{k} \hat{f}(k)\right\|_{B_{p, q}^{s}} \leq C\|f\|_{B_{p, q}^{s}} .
$$

Let $x \in X$ and define $f(t)=e_{n} \otimes x$ for $n \in \mathbb{Z}$ fixed. Then the above inequality implies $\left\|e_{n}\right\|_{B_{p, q}^{s}}\left\|M_{n} x\right\|=\left\|e_{n} M_{n} x\right\| \leq C\left\|e_{n}\right\|_{B_{p, q}^{s}}\|x\|$. Hence $\left\|M_{n}\right\| \leq C$

$($ ii $) \Rightarrow(i)$. From the proof of Proposition 2.8 we have the identity

$$
k\left(M_{k+1}-M_{k}\right)=\frac{k\left(a_{k+1}-a_{k}\right)}{a_{k}}\left(I-a_{k+1} A\right)^{-1}\left[\left(I-a_{k} A\right)^{-1}-I\right] .
$$


Observe that $\left\{a_{k}\right\}$ is 1-regular if and only if $\left\{\frac{1}{a_{k}}\right\}$ is 1-regular. Hence, boundedness of $\left\{M_{k}\right\}$ together with 1-regularity of $\left\{b_{k}\right\}$, imply that

$$
\sup _{k}\left\|k\left(M_{k+1}-M_{k}\right)\right\|<\infty .
$$

In order to verify the second condition, we observe that for $\lambda, \mu, \gamma \in \rho(A)$ we have the identity

$$
\begin{aligned}
\lambda R(\lambda, A) & -2 \mu R(\mu, A)+\gamma R(\gamma, A) \\
& =\mu R(\mu, A)\left[\frac{(\mu-\lambda)}{\mu} \frac{(\gamma-\lambda)}{\lambda}\right] \lambda R(\lambda, A)[\gamma R(\gamma, A)-I] \\
& -\mu R(\mu, A) \frac{(\lambda-2 \mu+\gamma)}{\mu}[\gamma R(\gamma, A)-I] .
\end{aligned}
$$

where $R(\lambda, A)=(\lambda-A)^{-1}$ denotes the resolvent operator. Note that $M_{k}=$ $b_{k} R\left(b_{k}, A\right)$. Substituting $\lambda=b_{k-1}, \mu=b_{k}, \gamma=b_{k+1}$, we obtain

$$
\begin{aligned}
k^{2}\left(M_{k-1}\right. & \left.-2 M_{k}+M_{k+1}\right) \\
& =M_{k}\left[\frac{k\left(b_{k}-b_{k-1}\right)}{b_{k-1}} \frac{k\left(b_{k+1}-b_{k-1}\right)}{b_{k}}\right] M_{k-1}\left[M_{k+1}-I\right] \\
& -M_{k} \frac{k^{2}\left(b_{k-1}-2 b_{k}+b_{k+1}\right)}{b_{k}}\left[M_{k+1}-I\right] .
\end{aligned}
$$

Since $\left\{b_{k}\right\}$ is 1-regular, we get that $\alpha_{k}:=\frac{k\left(b_{k}-b_{k-1}\right)}{b_{k-1}}$ and $\beta_{k}:=\frac{k\left(b_{k+1}-b_{k-1}\right)}{b_{k}}$ are bounded. In fact, let $m_{k}:=\frac{k\left(b_{k+1}-b_{k}\right)}{b_{k}}$, then we have

$$
\alpha_{k}=m_{k-1}+\frac{\left(b_{k}-b_{k-1}\right)}{b_{k-1}}
$$

and

$$
\beta_{k}=m_{k}+m_{k-1} \frac{b_{k-1}}{b_{k}}
$$

It follows from $\left|k\left(\frac{b_{k+1}}{b_{k}}-1\right)\right| \leq C$ for some $C>0$ that $\left|\frac{b_{k+1}}{b_{k}}-1\right| \leq \frac{C}{|k|}$ and thus $\frac{b_{k+1}}{b_{k}} \rightarrow 1$, proving the claim. 
On the other hand, by 2-regularity, $\frac{k^{2}\left(b_{k-1}-2 b_{k}+b_{k+1}\right)}{b_{k}}$ is bounded. Hence

$$
\sup _{k}\left\|k^{2}\left(M_{k-1}-2 M_{k}+M_{k+1}\right)\right\|<\infty
$$

and the result follows from Theorem 3.3.

Definition 3.5. Let $1 \leq p, q \leq \infty$ and $s>0$. A function $u \in B_{p, q}^{s+1}(\mathbb{T} ; X)$ is called a strong $B_{p, q}^{s}$ - solution of (1.1) if $u(t) \in D(A)$ and (1.1) holds for a.e. $t \in[0,2 \pi]$

Remark 3.6.

By [5, Theorem 1.3] if $u$ is a strong $B_{p, q}^{s}$ - solution of (1.1), then $u$ is differentiable a.e. and $u^{\prime} \in B_{p, q}^{s}(\mathbb{T} ; X)$.

We recall from Section 2 the notation

$$
\begin{aligned}
c_{k} & =\tilde{a}(i k) ; \\
b_{k} & =\frac{1}{a_{k}}=\frac{i k}{1+\tilde{a}(i k)} \text { for all } k \in \mathbb{Z} \backslash\{0\} ; \quad b_{0}=0 .
\end{aligned}
$$

We recall also that $(a \dot{*} u)(t)=\int_{-\infty}^{t} a(t-s) u(s) d s$.

We introduce the following condition

(H2) $\left\{k c_{k}\right\}$ and $\left\{k^{2}\left(c_{k+1}-2 c_{k}+c_{k+1}\right)\right\}$ are bounded sequences.

\section{Remark 3.7.}

(1) The case $a(t)=\chi_{[0, T]}(t)$ already considered above (Example 2.16) in which $\tilde{a}(\lambda)=\frac{1-e^{-\lambda T}}{\lambda}, \tilde{a}(0)=T$, does not in general satisfy condition 
$(H 2)$. However, if $T=2 \pi$, we have $\tilde{a}(i k)=0, k \in \mathbb{Z} \backslash\{0\}$ so that (H2) is trivially satisfied. Note that in this case, $\tilde{a}(\lambda)$ does not satisfy the conditions of the Analytic Representation Theorem ([2, Theorem 2.6.1]). It does not satisfy the condition imposed by Da Prato-Lunardi ([12, Condition(5.2), p.104]) either.

(2) One verifies that for $a(t)=b e^{-c t},(c>0),(H 2)$ is satisfied. More generally, if $n \in \mathbb{N}$ and $a(t)=\frac{t^{n}}{(n-1) !} e^{-c t}($ where $c>0)$ then $\tilde{a}(\lambda)=$ $\frac{1}{(\lambda+c)^{n}}$ and for $n \geq 2,(H 2)$ is trivially satisfied since $k^{2} c_{k}=\frac{k^{2}}{(c+i k)^{n}}$ is bounded.

In the following lemma we show, in particular, that condition $(H 2)$ implies condition $(H 1)$.

Lemma 3.8. Let $X$ be a Banach space. We have

(i) If $\left\{k c_{k}\right\}$ is bounded, then condition (H1) holds. Moreover,

(ii) under condition (H2), the sequences $\left\{\left(1+c_{k}\right) I\right\}_{k \in \mathbb{Z}},\left\{\frac{\left(1+c_{k}\right)}{i k} I\right\}_{k \in \mathbb{Z} \backslash\{0\}}$ and $\left\{\left(\frac{1}{1+c_{k}}\right) I\right\}_{k \in \mathbb{Z}}$ are $B_{p, q}^{s}(0,2 \pi ; X)$-multipliers.

Proof. (i) Clearly, condition (H2) implies that $\left\{c_{k}\right\}$ is bounded. Hence $k\left(c_{k+1}-c_{k}\right)=(k+1) c_{k+1}-c_{k+1}-k c_{k}$ is bounded and $\lim _{k \rightarrow \infty} c_{k}=0$. This proves that $\frac{1}{1+c_{k}}$ is also bounded and (H1) is satisfied.

(ii) By (i), and hence $(H 1)$, the sequence $\left\{m_{k}:=\left(1+c_{k}\right) I\right\}_{k \in \mathbb{Z}}$ is bounded and satisfies

$$
k\left(m_{k+1}-m_{k}\right)=k \frac{\left(c_{k}-c_{k+1}\right)}{\left(1+c_{k+1}\right)\left(1+c_{k}\right)}
$$

By $(H 2)$, we obtain directly that $\left\{k^{2}\left(m_{k+1}-2 m_{k}+m_{k-1}\right)\right\}$ is also bounded and hence $\left\{m_{k}\right\}$ is a $B_{p, q}^{s}$-multiplier. 
For $a_{k}=\frac{1+c_{k}}{i k}$ we obtain the identity

$$
\begin{aligned}
k^{2}\left(a_{k+1}-2 a_{k}+a_{k-1}\right) & =\frac{k^{2}}{i(k+1) k(k-1)}\left[k^{2}\left(c_{k+1}-2 c_{k}+c_{k-1}\right)\right. \\
& \left.-k\left(c_{k+1}-c_{k-1}\right)+2\left(1+c_{k}\right)\right],
\end{aligned}
$$

and therefore $k^{2}\left(a_{k+1}-2 a_{k}+a_{k-1}\right)$ is bounded, which proves that $\left\{a_{k}\right\}$ is a $B_{p, q}^{s}$-multiplier. Finally, if $n_{k}:=\frac{1}{1+c_{k}}$ then

$$
\begin{aligned}
k^{2}\left(n_{k+1}-2 n_{k}+n_{k-1}\right) & =\frac{-k^{2}\left(c_{k-1}-2 c_{k}+c_{k+1}\right)}{\left(1+c_{k}\right)\left(1+c_{k+1}\right)\left(1+c_{k-1}\right)} \\
& -\frac{k c_{k-1} k\left(c_{k+1}-c_{k}\right)}{\left(1+c_{k}\right)\left(1+c_{k+1}\right)\left(1+c_{k-1}\right)} \\
& +\frac{k c_{k+1} k\left(c_{k}-c_{k-1}\right)}{\left(1+c_{k}\right)\left(1+c_{k+1}\right)\left(1+c_{k-1}\right)}
\end{aligned}
$$

and hence $k^{2}\left(n_{k+1}-2 n_{k}+n_{k-1}\right)$ is bounded, showing that $\left\{n_{k}\right\}$ is also a $B_{p, q}^{s}$-multiplier.

Theorem 3.9. Let $1 \leq p, q \leq \infty$ and $s>0$. Let $A$ be a closed linear operator defined on a Banach space $X$ and assume that condition (H2) is satisfied and $\left\{b_{k}\right\}$ is 2-regular. The following assertions are equivalent:

(i) $\left\{b_{k}\right\}_{k \in \mathbb{Z}} \subset \rho(A)$ and $\sup _{k}\left\|b_{k}\left(b_{k} I-A\right)^{-1}\right\|<\infty$.

(ii) For every $f \in B_{p, q}^{s}(\mathbb{T} ; X)$ there exists a unique strong $B_{p, q}^{s}$ - solution of (1.1) such that $u^{\prime}, A u$ and $a \dot{*} A u \in B_{p, q}^{s}(\mathbb{T} ; X)$.

Proof. $\quad(i i) \Rightarrow(i)$. Let $x \in X$ be fixed. Let $k \in \mathbb{Z}$ and let $f(t)=e_{k} \otimes x$. Note that $f \in B_{p, q}^{s}(\mathbb{T} ; X)$. Hence there exists $u \in B_{p, q}^{s+1}(\mathbb{T} ; X)$ such that $u(t) \in D(A)$ and (1.1)holds for a.e. $t \in[0,2 \pi]$. Following the same reasoning as in the proof of Theorem 2.12 we obtain that $\left\{b_{k}\right\}_{k \in \mathbb{Z}} \subset \rho(A)$. 
By hypothesis, and using the Closed Graph Theorem, we obtain that there exists $C>0$ independent of $f$ and $u$ such that

$$
\|u\|_{B_{p, q}^{s+1}}+\|A u\|_{B_{p, q}^{s}}+\|a \dot{*} A u\|_{B_{p, q}^{s}} \leq C\|f\|_{B_{p, q}^{s}} .
$$

We consider $f(t)=e_{k} \otimes x$ for some $k \in \mathbb{Z}$ and $x \in X$. The solution $u$ satisfies $u^{\prime}(t)=b_{k}\left(b_{k} I-A\right)^{-1} e_{k} \otimes x$ (see equation (2.5)). The above estimate implies that

$$
\left\|b_{k}\left(b_{k} I-A\right)^{-1} x\right\| \leq C\|x\| \text { for all } k \in \mathbb{Z} .
$$

This proves the implication.

$(i) \Rightarrow(i i)$. Because of Lemma 3.8, the proof follows the same lines as the proof of Theorem 2.12 in section 2. We omit the details.

In case $p=q=\infty$ and $0<s<1$ we have $B_{\infty, \infty}^{s}(\mathbb{T} ; X)$ corresponds to the space $C^{s}(\mathbb{T} ; X)$ of Hölder continuous functions (see e.g. [4]). We state the corresponding result separately:

Theorem 3.10. Let $A$ be a closed linear operator defined on a Banach space $X$ and let $0<\alpha<1$. Assume that condition (H2) is satisfied and $\left\{b_{k}\right\}$ is 2-regular. The following assertions are equivalent:

(i) $\left\{b_{k}\right\}_{k \in \mathbb{Z}} \subset \rho(A)$ and $\sup _{k}\left\|b_{k}\left(b_{k} I-A\right)^{-1}\right\|<\infty$.

(ii) For every $f \in C^{\alpha}(0,2 \pi ; X)$ there exists a unique strong $C^{\alpha}$ - solution of (1.1) such that $u^{\prime}, A u$ and $a \dot{*} A u \in C^{\alpha}(0,2 \pi ; X)$.

We remark that if $X$ is $U M D$ space then condition $(H 1)$ is enough for the validity of Theorem 3.9 and Theorem 3.10. Moreover we do not need to 
assume that the sequence $\left(b_{k}\right)$ is 2-regular. This follows from [5, Theorem $3.2]$.

Theorem 3.9 and Theorem 3.10 apply in particular when $A$ generates a bounded holomorphic semigroup on $X$. This case is studied in Da PratoLunardi $[11,12]$ where the semigroup is used for a direct construction of the solution of the equation.

Acknowledgements: The authors wish to thank the referee for useful suggestions and for pointing out to them the reference [14].

\section{REFERENCES}

[1] H. Amann. Linear and Quasilinear Parabolic Problems. Monographs in Mathematics. 89. Basel: Birkhäuser Verlag, 1995.

[2] W. Arendt, C.J.K. Batty, M Hieber and F. Neubrander. Vector-valued Laplace Transforms and Cauchy Problems. Monographs in Mathematics. 96. Basel: Birkhäuser Verlag, 2001.

[3] W. Arendt, S. Bu. The operator-valued Marcinkiewicz multiplier theorem and maximal regularity. Math. Z. 240(2002), 311-343.

[4] W. Arendt, C. Batty, S. Bu. Fourier multipliers for Hölder continuous functions and maximal regularity. Ulmer Seminare 2001, Heft 6 (2001), 1-25.

[5] W. Arendt, S. Bu. Operator-valued Fourier multipliers on periodic Besov spaces and applications.Ulmer Seminare 2001, Heft 6 (2001), 26-41.

[6] J. Bourgain. Vector-valued singular integrals and the $H^{1}-B M O$ duality. Probability Theory and Harmonic Analysis, Marcel Dekker, New York, 1986.

[7] P.L. Butzer, H. Berens, Semi-Groups of Operators and Approximation. Die Grundlehren der Mathematische Wissenschaften, 145, Springer Verlag, 1967.

[8] B.D. Coleman, M.E. Gurtin, Equipresence and constitutive equation for rigid heat conductors. Z. Angew. Math. Phys. 18(1967), 199-208. 
[9] Ph. Clément, G. Da Prato. Existence and regularity results for an integral equation with infinite delay in a Banach space. Integral Equations Operator Theory, 11(1988), 480-500.

[10] Ph. Clément, B. de Pagter, F. A. Sukochev, M. Witvliet. Schauder decomposition and multiplier theorems. Studia Math. 138(2000), 135-163.

[11] G. Da Prato, A. Lunardi. Periodic solutions for linear integrodifferential equations with infinite delay in Banach spaces. Differential Equations in Banach spaces. Lecture Notes in Math. 1223(1985), 49-60.

[12] G. Da Prato, A. Lunardi. Solvability on the real line of a class of linear Volterra integrodifferential equations of parabolic type. Ann. Mat. Pura Appl. 4(1988), 67-117.

[13] B. De Pagter, H. Witvliet. Unconditional decompositions and UMD spaces. Publ. Math. Besançon, Fasc. 16(1998), 79-111.

[14] Fašangová, E., Prüss, J. Asymptotic behaviour of a semilinear viscoelastic beam model. Arch. Math. (Basel) 77(2001),488-497.

[15] J.W. Nunziato. On heat conduction in materials with memory. Quart. Appl. Math. 29(1971), 187-304.

[16] J. Prüss. Evolutionary Integral Equations and Applications. Monographs Math., vol. 87, Birkhäuser Verlag, 1993.

[17] H. Triebel. Theory of Function Spaces. Monographs Math., vol. 78, Birkhäuser Verlag, Basel, 1983.

[18] L. Weis. Operator-valued Fourier multiplier theorems and maximal $L_{p}$-regularity. Math. Ann. 319 (2001), 735-758.

[19] L. Weis. A new approach to maximal $L_{p}$-regularity. Lect. Notes Pure Appl. Math. 215, Marcel Dekker, New York, (2001), 195-214. 
University of Puerto Rico, Department of Mathematics and Computer Science, Faculty of Natural Sciences, PO Box 23355, Puerto Rico - 00931 U.S.A.

E-mail address: keyantuo@rrpac.upr.clu.edu

Universidad de Santiago de Chile, Departamento de Matemática, Facultad de Ciencias, Casilla 307-Correo 2, Santiago-Chile.

E-mail address: clizama@usach.cl 